Proceedings of the 1992 IEEE

Intemational Conference on Robotics and Automation

Nice, France - May 1992

\title{
Finding Antipodal Point Grasps on Irregularly Shaped Objects
}

\author{
I-Ming Chen, Joel W. Burdick \\ School of Engineering and Applied Science \\ California Institute of Technology \\ Pasadena, CA 91125
}

\begin{abstract}
This paper considers two finger antipodal point grasping of arbitrarily shaped 2-D and 3-D objects. We introduce an object function which maps a finger contact space to the object surface. Conditions are developed to identify the feasible grasping region, $\mathcal{F}$, in the finger contact space. We introduce a "grasping energy function," $E$, which is proportional to the distance between two grasping points. The antipodal points correspond to critical points of $E$ in $\mathcal{F}$. Optimization and/or continuation techniques are used to find these critical points. In particular, we apply global optimization techniques to find the "maximal grasp". Further, we introduce modelling techniques for representing 2$\mathrm{D}$ and 3-D objects using B-spline curves and spherical product surfaces.
\end{abstract}

\section{Introduction}

This paper considers two-finger antipodal point grasps. Antipodal points on an object have surface normal vectors which are collinear and opposite in sense. With appropriate finger contact conditions, antipodal point grasps guarantee force closure. We address two issues: (1) how to describe natural and man-made objects with smooth boundaries; and (2) how to find the antipodal point pairs on such objects. We also consider the problem of finding the maximal grasp, which is the pair of antipodal points which are maximally separated on the object surface.

Grasp synthesis for polygonal objects has been considered by several authors, including [10,12]. Antipodal points on smooth objects are also considered in [7] in order to implement a "finger gait" form of object manipulation. However no explicit algorithm is given to find them. We note that in [6], Faverjon finds the independent grasp region for force closure grasp by using a cell decomposition technique. However, the maximal grasp or the antipodal point grasps may not lie in the maximal grasp rectangle, and this paper did not consider expanding grasps.

To find antipodal points on smooth objects, we define a grasping energy function which is proportional to the distance between the two fingertips. The critical points of this function which lie in the feasible grasping region in the finger contact space correspond to pairs of antipodal points. The energy maximum corresponds to the maximal grasp. To model natural and man-made objects we introduce 2-D and 3-D representation systems based on spline functions and spherical product surfaces. We demonstrate with examples the application of our scheme to objects modelled with this representation system. A more complete exposition can be found in [5].

\section{Antipodal Point Grasps on 3-D Objects}

\subsection{Two-Dimensional Object Functions}

Assuming that a planar object is smooth and devoid of holes, its closed boundary curve can be described or closely approximated by a parametric curve $[x(s), y(s)]$, where $s \in I_{s}=\left[s_{0}, s_{1}\right]$.

Definition 2.1 A 2-dimensional object function $\mathcal{O}_{2 D}$ $I_{d} \rightarrow R^{2}$ is defined by:

$$
\mathcal{O}_{2 D}(s)=[x(s), y(s)], \quad \forall s \in I_{s}
$$

Since the object is smooth, $x^{\prime 2}(s)+y^{\prime 2}(s)>0, \forall s \in I_{s}$, where $x^{\prime} \equiv \frac{d x}{d s}$ and $y^{\prime} \equiv \frac{d y}{d \theta}$. The unit tangent vector on $\mathcal{O}_{2 D}$ is given by $\mathrm{t}=\left[x^{\prime}(s), y^{\prime}(s)\right] /\left[x^{\prime 2}(s)+{y^{\prime 2}}^{2}(s)\right]^{1 / 2}$. The outward normal vector $\mathbf{n}$ has two different forms depending on the direction of parametrization. Let $\|\mathrm{n}\|=\left[x^{\prime 2}(s)+y^{\prime 2}(s)\right]^{1 / 2}$, then

$$
\mathbf{n}= \begin{cases}{\left[-y^{\prime}(s), x^{\prime}(s)\right] /\|\mathbf{n}\|,} & \text { for } s \text { clockwise } \\ {\left[y^{\prime}(s),-x^{\prime}(s)\right] /\|\mathbf{n}\|,} & \text { for } s \text { counter clockwise. }\end{cases}
$$

Denote the two finger contact points on $\mathcal{O}_{2 D}$ by $\mathbf{p}_{1} \equiv$ $\mathcal{O}_{2 D}\left(s_{1}\right)$ and $p_{2} \equiv \mathcal{O}_{2 D}\left(s_{2}\right)$. The ordered pair $\left(s_{1}, s_{2}\right)$, termed a grasp configuration in [6], represents a twofinger grasp on object $\mathcal{O}_{2 D}(s)$.

Definition 2.2: The planar finger contact space, $\mathcal{C}_{2}$, of the object $\mathcal{O}_{2 D}: I_{A} \rightarrow R^{2}$ is the Cartesian product of the domain of $\mathcal{O}_{2 D}$. Typically, $\mathcal{C}_{2} \equiv I_{s} \times I_{s}$.

$\mathcal{C}_{2}$ contains all possible 2-finger grasp configurations.

2.2 The Feasible Grasping Region of a 2-D Object

Let $\mathbf{p}$ and $\mathbf{p}_{2}$ denote the two points on the object surface corresponding to $\left(s_{1}, s_{2}\right)$. We assume that the two finger contacts are point contact with friction. Force closure is achieved at $\left(s_{1}, s_{2}\right)$ iff the line segment $\overline{p_{1} p_{2}}$ lies in the friction cones at $p_{1}$ and $p_{2}$ [11].

A friction cone, $\mathcal{S}$, at contact $p$ consists of two sectors: one extending outside of the object is called the positive friction cone $\mathcal{S}^{+}$, the other extending in- 


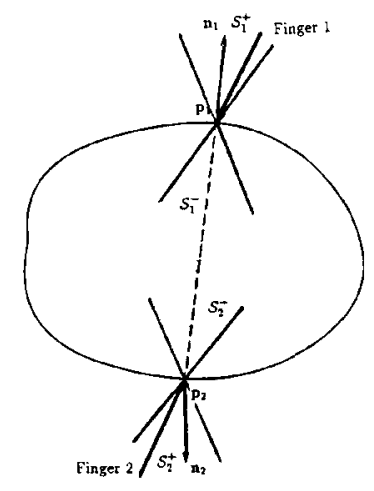

squeezing

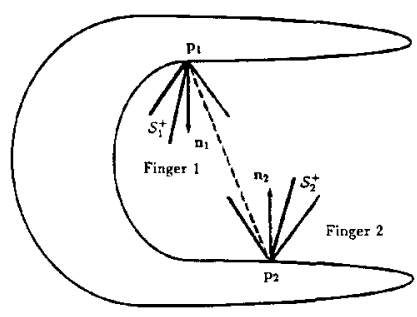

expanding

Figure 1: Force closure grasps

side the object is called the negative friction cone $\mathcal{S}^{-}$ (fig.1). Both squeezing grasps, where $\overline{\mathbf{p}_{1} \mathbf{p}_{2}}$ falls inside $\mathcal{S}_{1}^{-}$and $\mathcal{S}_{2}^{-}$, and expanding grasps, where $\overline{\mathbf{p}_{1} \mathrm{p}_{2}}$ lies in $\mathcal{S}_{1}^{+}$ and $S_{2}^{+}$, satisfy the force closure condition. A convex body can be grasped by squeezing grasp only. Nonconvex bodies can admit both squeering and expanding grasps.

Assume that the friction coefficient, $\mu$, is uniform on $\mathcal{O}_{2 D}$ and denote $c_{f}=\cos \left(\tan ^{-1} \mu\right)$. Let $n_{1}$ and $n_{2}$ be the normal vectors at $p_{1}$ and $p_{2}$. The conditions for a squeezing grasp are:

$$
\begin{aligned}
& f_{1}^{-}\left(s_{1}, s_{2}\right)=\mathbf{n}_{1} \cdot\left(\mathbf{p}_{1}-\mathbf{p}_{2}\right)-c_{f}\left\|\mathbf{p}_{1}-\mathbf{p}_{2}\right\|>0 \\
& f_{2}^{-}\left(s_{1}, s_{2}\right)=\mathbf{n}_{2} \cdot\left(\mathbf{p}_{2}-\mathbf{p}_{1}\right)-c_{f}\left\|\mathbf{p}_{2}-\mathbf{p}_{1}\right\|>0
\end{aligned}
$$

The conditions for an expanding grasp are:

$$
\begin{aligned}
& f_{1}^{+}\left(s_{1}, s_{2}\right)=\mathbf{n}_{1} \cdot\left(\mathbf{p}_{1}-\mathbf{p}_{2}\right)+c_{f}\left\|\mathbf{p}_{1}-\mathbf{p}_{2}\right\|<0(2.4) \\
& f_{2}^{+}\left(s_{1}, s_{2}\right)=\mathbf{n}_{2} \cdot\left(\mathbf{p}_{2}-\mathbf{p}_{1}\right)+c_{f}\left\|\mathbf{p}_{2}-\mathbf{p}_{1}\right\|<0 .(2.5) \\
& \text { Let } \mathcal{F}^{-}=\left\{\left(s_{1}, s_{2}\right) \mid f_{1}^{-}>0, f_{2}^{-}>0 ;\left(s_{1}, s_{2}\right) \in \mathcal{C}_{2}\right\} \\
& \mathcal{F}^{+}=\left\{\left(s_{1}, s_{2}\right) \mid f_{1}^{+}<0, f_{2}^{+}<0 ;\left(s_{1}, s_{2}\right) \in \mathcal{C}_{2}\right\},
\end{aligned}
$$

$\mathcal{F}^{-}$represents all squeezing force closure grasps and $\mathcal{F}^{+}$represents all expanding force closure grasps.

Definition 2.3 The 2-fingered feasible grasping region, $\mathcal{F}$, for $\mathcal{O}_{2 D}$ is the set $\mathcal{F}=\mathcal{F}^{-} \cup \mathcal{F}^{+}$in $\mathcal{C}_{2}$ containing

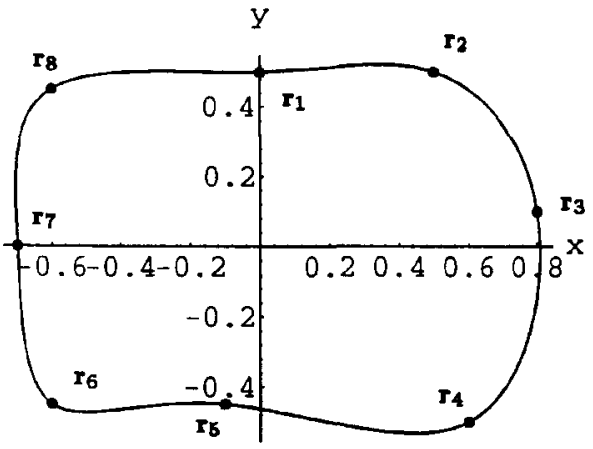

Figure 2: A 2-D object function

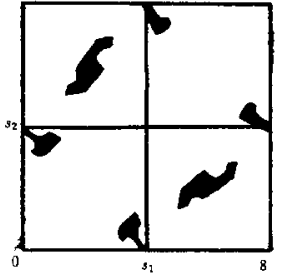

$\mu=0.2$

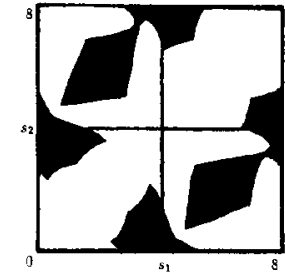

$\mu=0.8$
Figure 3: The feasible grasping regions

all force closure grasp configurations in $\mathcal{C}_{2}$. Any grasp configuration in $\mathcal{F}$ admits a two-finger force closure grasp.

The geometry of $\mathcal{F}$ is a function of $\mathcal{O}_{2 D}$ and $\mu$. $\mu$ might be a function of the finger tip location, $\mu=\mu(s)$. Here we assume $\mu$ is a constant. The feasible grasping regions for the object in fig.2 are shown in fig. 3 for $\mu=$ $0.2,0.8$ respectively. As $\mu \rightarrow 0, \mathcal{F}$ shrinks to isolated points, lines, or patches which correspond to antipodal points. Antipodal point grasps remain force closure as $\mu \rightarrow 0$, and are thus robust with respect to variations of $\mu$. Note that $\mathcal{F}$ is symmetric with respect to the $s_{1}=s_{2}$ line since $\left(s_{1}, s_{2}\right)$ and $\left(s_{2}, s_{1}\right)$ have the same force closure characteristics.

\subsection{Antipodal Points}

A pair of antipodal points $p_{1}$ and $p_{2}$ on an object satisfy the following conditions:

$$
\begin{array}{r}
\left(\mathbf{p}_{1}-\mathbf{p}_{2}\right) \cdot \mathbf{t}_{1}=0 \\
\left(\mathbf{p}_{2}-\mathbf{p}_{1}\right) \cdot \mathbf{t}_{2}=0 \\
\mathbf{n}_{1}+\mathbf{n}_{2}=0
\end{array}
$$

where $t_{1}, t_{2}$ and $n_{1}, n_{2}$ are the unit tangent vectors and outward normal vectors at $\mathbf{p}_{1}, \mathbf{p}_{2}$ respectively.

\subsection{A Grasping Energy Function}

A grasping energy function $E: C_{2} \rightarrow R$, which is proportional to the square of the length of $\overline{p_{1} p_{2}}$, is defined as: 


$$
\begin{aligned}
E\left(s_{1}, s_{2}\right) & =\frac{1}{2} \kappa\left\|p_{1}-p_{2}\right\|^{2} \\
& =\frac{1}{2} \kappa\left\|\mathcal{O}_{2 D}\left(s_{1}\right)-\mathcal{O}_{2 D}\left(s_{2}\right)\right\|^{2}
\end{aligned}
$$

$E$ can be interpreted as the energy of a spring, with spring constant $\kappa$, connecting $\mathbf{p}_{1}$ and $\mathbf{p}_{\mathbf{2}}$.

Proposition 2.1 A pair of antipodal points, $p_{1}$ and $\mathrm{p}_{2}$, on $\mathcal{O}_{2 D}$ corresponds to a critical point of $E$. Conversely, a critical point of $E$ lying in $\mathcal{F}$ corresponds to a pair of antipodal points on $\mathcal{O}_{2 D}$. The maximum of $E$ is the maximal grasp.

Proof: Let $\mathbf{p}_{\mathbf{1}}$ and $\mathbf{p}_{\mathbf{2}}$ be antipodal points. The unit tangent vectors at $p_{1}, p_{2}$ are: $\mathbf{t}_{i}=\mathcal{O}_{2} D^{\prime}\left(s_{i}\right) /\left\|\mathcal{O}_{2} D^{\prime}\left(s_{i}\right)\right\|$, $(i=1,2)$. Substituting $p_{1}, p_{2}, t_{1}$, and $t_{2}$ into (2.6-2.8), we get:

$$
\begin{aligned}
& {\left[\mathcal{O}_{2 D}\left(s_{1}\right)-\mathcal{O}_{2 D}\left(s_{2}\right)\right] \cdot \frac{\mathcal{O}_{2 D}\left(s_{1}\right)}{\left\|\mathcal{O}_{2 D}{ }^{\prime}\left(s_{1}\right)\right\|}=0} \\
& {\left[\mathcal{O}_{2 D}\left(s_{2}\right)-\mathcal{O}_{2 D}\left(s_{1}\right)\right] \cdot \frac{\mathcal{O}_{2 D}\left(s_{2}\right)}{\left\|\mathcal{O}_{2} D^{\prime}\left(s_{2}\right)\right\|}=0} \\
& {\left[-y^{\prime}\left(s_{1}\right), x^{\prime}\left(s_{1}\right)\right]+\left[-y^{\prime}\left(s_{2}\right), x^{\prime}\left(s_{2}\right)\right]=0 .}
\end{aligned}
$$

Let $s_{c}=\left(s_{1 c}, s_{2}\right)$ be a critical point of $E$. $s_{c}$ satisfies:

$$
\begin{aligned}
& \frac{\partial E}{\partial s_{1}}=\kappa\left[\mathcal{O}_{2 D}\left(s_{1}\right)-\mathcal{O}_{2 D}\left(s_{2}\right)\right] \cdot \mathcal{O}_{2 D}{ }^{\prime}\left(s_{1}\right)=0 \\
& \frac{\partial E}{\partial s_{2}}=\kappa\left[\mathcal{O}_{2 D}\left(s_{2}\right)-\mathcal{O}_{2 D}\left(s_{1}\right)\right] \cdot \mathcal{O}_{2 D}\left(s_{2}\right)=0 .
\end{aligned}
$$

Antipodal points $p_{1}$ and $p_{2}$ satisfy (2.13) and (2.14), and are therefore critical points of $E$. Conversely, a critical point of $E$ does not necessarily satisfy (2.8) or (2.12). If $s_{c}$ lies in $\mathcal{F}$, the corresponding grasp points $\mathbf{p}_{1}, \mathbf{p}_{2}$ and unit outward normal $\mathbf{n}_{1}, \mathbf{n}_{2}$ will satisfy $\mathbf{n}_{1} \cdot \frac{\mathbf{p}_{1}-\mathbf{p}_{2}}{\left\|\mathbf{P}_{1}-\mathbf{p}_{2}\right\|}=1$ and $\mathbf{n}_{2} \cdot \frac{\mathbf{p}_{2}-\mathbf{p}_{1}}{\left\|\mathbf{P}_{2}-\mathbf{p}_{1}\right\|}=1$ (or both equal to -1), which implies that $\mathbf{n}_{1}$ and $\mathbf{n}_{2}$ are collinear and in opposite direction. Hence (2.8) holds and $s_{c}$ represents a pair of antipodal points. Since $E$ is differentiable in the compact domain $\mathcal{C}_{2}, E$ must achieve a maximum, $s_{m}=\left(s_{m 1}, s_{m 2}\right)$, in $C_{2}$. It can be shown [5] that $s_{m}$ is a point of antipodal points corresponding to the maximal grasp.|

The nature of the critical points of $E$ depend upon the local geometry of the object near the antipodal points. Let $\left(s_{1}^{*}, s_{2}^{*}\right)$ denote an antipodal point pair. If $\mathcal{O}_{2 D}$ is convex at $s_{1}^{*}$ and convex at $s_{2}^{*}, E\left(s_{1}^{*}, s_{2}^{*}\right)$ will be a local maximum. If the object is concave at both $s_{1}^{*}$ and $s_{2}^{*}$, the critical point will be a local minimum. If the object is convex at one antipodal point and concave at the other, the critical point may be a saddle point or a local minimum, depending on the relative local curvatures of the object function at $s_{1}^{*}$ and $s_{2}^{*}$. The maximally separated antipodal points are necessarily locally convex.

Proposition 2.1 suggests that antipodal points can be found by optimization schemes or other methods for finding critical points of a function. In particular, the maximal grasp can be formulated as the constrained global optimization problem: maximize

subject to

$$
\begin{aligned}
& E\left(s_{1}, s_{2}\right)=\frac{1}{2} \kappa\left\|\mathcal{O}_{2 D}\left(s_{1}\right)-\mathcal{O}_{2 D}\left(s_{2}\right)\right\|^{2} \\
& \left(s_{1}, s_{2}\right) \in \mathcal{F}
\end{aligned}
$$

Note that $\kappa$ can be adjusted to alter the speed of convergence in the numerical critical point finding process.

\section{Methods for Finding the Critical Points of $E$}

Numerical techniques which find the critical points of $E$ are required to find antipodal points. Practically, we are more interested in finding the subset of critical points which are local and global maxima of $E$. These points correspond to antipodal points with local object convexity. In grasp planning, such points typically do not require additional calculations which check for geometric interference between the grasping fingers and the object surface.

Any suitable constrained optimization method can be used to find local maxima of $E$ in $\mathcal{F}$ [2]. The antipodal points found by these local methods will strongly depend on the procedure's initial conditions. Multiple random start methods [3] can be used to find all of the local critical points. Constrained global optimization techniques [8] can be used to find the global maximum of $E$ corresponding to a maximal grasp. We use a recently developed global optimization algorithm, termed TRUST [4]. TRUST uses a novel "tunneling" method which finds the global extrema by repeatedly escaping local extrema. Thus, on the way to finding the global solution, many local critical points, which correspond to feasible, but non-maximal, antipodal grasping points, are also identified.

In practice, portions of the object surface may be occluded by nearby objects, or not visible to a robot vision system which generates object models. In such cases, additional constraints can be added to the optimizaton procedure to exclude these regions of the surface in the optimization process.

Maximization methods will miss antipodal points which are saddle points or local minima of $E$. Local minima can easily be found by maximization of $-E$. All critical points of $E$ can be found using continuation techniques [9]. These techniques are useful when the critical points of $E$ do not form isolated sets, which occurs when the object contains parallel faces.

\section{Representations of 2-Dimensional Objects}

We will use cubic B-spline curves to represent irregularly shaped objects. This modelling method is computationally efficient, produces surfaces with satisfactory smoothness ( $C^{2}$ continuity, which is required for many numerical schemes), and handles certain straight lines and sharp corners [13]. A B-spline curve is a collection of piecewise continuous cubic polynomial curve segments. If the parameter intervals for every segments are equal, we call it a uniform cubic $B$-spline curve. A uniform B-spline curve of $n$ segments is the collection of curve segments having the form:

$$
q_{i}(t)=a_{i} t^{3}+b_{i} t^{2}+c_{i} t+d_{i}
$$

where $a_{i}, b_{i}, c_{i}, d_{i} \in R^{2}, i=1, \cdots, n$, and $t$ is the curve segment parameter, $t \in I \equiv[0,1]$. Let s be a global curve parameter accumulating the values of 


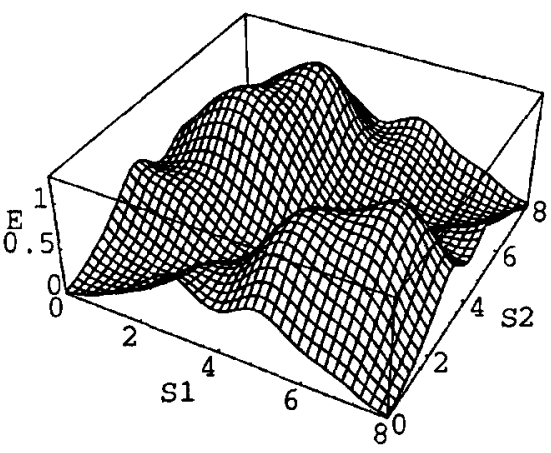

Figure 4: The energy function $E$

local parameters $t$ of each segment. For an $n$ segment curve, $s \in[0, n]$.

Unoccluded planar objects are modelled by closed cubic B-spline curves. The 2-dimensional object function $\mathcal{O}_{2 p}: I_{s} \equiv I_{n} \rightarrow R^{2}$ for an object which is modelled by a closed B-spline of $n$ segments is:

$$
\mathcal{O}_{2 D}(s)=[x(s), y(s)]=a(s) \hat{s}^{3}+b(s) \hat{s}^{2}+c(s) \hat{s}+d(s)
$$

where $\hat{s}=a-\lfloor s\rfloor, 0 \leq s \leq n,(\lfloor s\rfloor=n \Leftrightarrow n \leq s<n+1)$, and $a(s)=\left[a_{x}(s), a_{y}(s)\right]=a_{i}$, if $: \in[i-1, i), i=1 \cdots n$; $a_{1}$, if $s=n$. The definitions of $b(s), c(s)$, and $d(s)$ follow. The constants $a_{i}, b_{i}$, etc., in the interval $i-1 \leq$ $s<i$ are the coefficients of the curve segment $q_{i}(t)$. In the closed curve case, the starting point must coincide with the ending point, i.e. $\mathcal{O}_{2 D}(0)=\mathcal{O}_{2 D c}(n)=\mathrm{d}_{1}$.

When parts of the object are occluded by nearby objects or unviewable from vision sensors, the object surface is modelled by one or more open B-spline curves. In the open curve case, the 2-dimensional object function $\mathcal{O}_{2 D}: I_{n} \rightarrow R^{2}$ has the same form as (4.2), only that $s=s-\lfloor s]$, if $0 \leq s<n ; 1$, if $s=n$. And $a(s)=a_{i}$, if $: \in[i-1, i), i=1 \ldots n_{n} ; a_{n}$, if $:=n$. The definitions of $b(s), c(s)$, and $d(s)$ follow. If an object is described by (4.2), $E$ will be $C^{2}$ continuous in $\mathcal{C}_{2}$, and Proposition 2.1 still holds.

The approximate boundary curve of an actual 2-D object can be obtained experimentally as follows. Data points which are uniform are used as the knot points first, and then the control points of the uniform cubic B-spline curve which describe the boundary can be calculated by an inversion process described in [13]. Data points not uniformly distributed on the object can be converted to a uniform basis by the addition more data points derived from the non-uniform model.

Example 1: The object in fig. 2 is assumed to be the approximation of a real object. The 8 real data points, $\mathbf{r}_{1} \ldots \mathbf{r}_{8}$, shown in the figure are the knot points of the B-spline curve. The energy function $E$ (where $\kappa=1$ ) is shown in fig. 4. Table 1 lists the global maximum and local extrema of $E$ found by TRUST. Since $E$ is symmetric to $s_{1}=s_{2}$ line, we list extrema with $s_{1}>s_{2}$ only. The corresponding maximal and antipodal point grasp locations are shown in fig. 5.

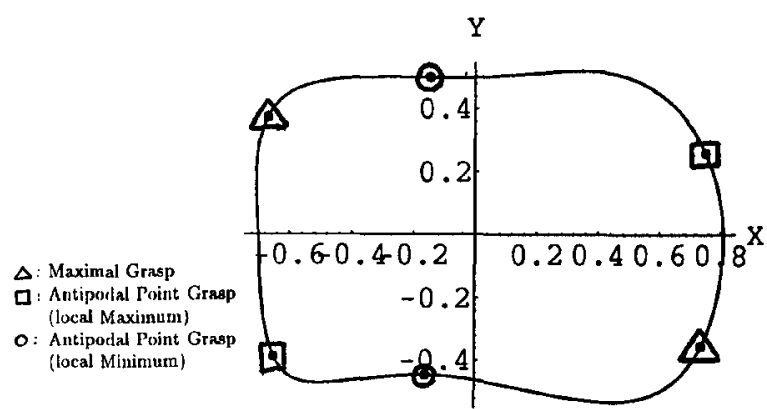

Figure 5: The maximal grasp and antipodal point grasps

\begin{tabular}{|c|c|r|c|}
\hline \multicolumn{4}{|c|}{ Table 1: Global and Local Extrema } \\
\hline Extrema & $\left(s_{1}, s_{2}\right)$ & $P_{1}(x, y)$ & $P_{2}(x, y)$ \\
\hline Global Max & $(6.75,2.67)$ & $(-0.67,0.37)$ & $(0.74,-0.35)$ \\
\hline Local Max & $(5.23,1.72)$ & $(-0.65,-0.39)$ & $(0.75,0.26)$ \\
\hline Local Min & $(7.78,4.09)$ & $(-0.14,0.50)$ & $(-0.16,-0.45)$ \\
\hline
\end{tabular}

\section{Antipodal Point Grasps on 3-D Objects}

This section extends the methodology for 2-D antipodal point grasping to 3-D grasping. We assume: a two-finger soft-contact model; that the object surface is smooth; and that $\mu$ is uniform on the object surface. With this two-finger contact model, force-closure is guaranteed if the line segment connecting the contact points lies in both of the negative (or positive) friction cones [11].

We assume that the 3-dimensional objects are devoid of holes, and thus are homeomorphic to a sphere.

Definition 5.1 A 3-dimensional object function $\mathcal{O}_{3 D}$ : $S^{2} \rightarrow R^{3}$ is defined by:

$$
\mathcal{O}_{3 D}(\mathrm{u})=[x(\mathrm{u}), y(\mathrm{u}), z(\mathrm{u})], \quad \mathrm{u} \in S^{2}
$$

The finger contact space of $\mathcal{O}_{3 D}$ is $\mathcal{C}_{3} \equiv S^{2} \times S^{2}$. Every $\left(\mathbf{u}_{1}, \mathbf{u}_{2}\right) \in \mathcal{C}_{3}$ represents a 2 -fingered grasp on $\mathcal{O}_{3 D}$.

The conditions for squeezing and expanding force closure grasps are similar to 2-dimensional case and bear the same form as (2.2-2.5). One can derive inequalities analogous to (2.2-2.5) which define feasible grasping regions: $g_{1}^{-}\left(\mathbf{u}_{1}, \mathbf{u}_{2}\right)>0, g_{2}^{-}\left(\mathbf{u}_{1}, \mathbf{u}_{2}\right)>0, g_{1}^{+}\left(\mathbf{u}_{1}, \mathbf{u}_{2}\right)<0$, and $g_{2}^{+}\left(\mathbf{u}_{1}, \mathbf{u}_{2}\right)<0$. Let

$$
\begin{aligned}
& \mathcal{G}^{-}=\left\{\left(\mathbf{u}_{1}, \mathbf{u}_{2}\right) \mid g_{1}^{-}>0, g_{2}^{-}>0 ;\left(\mathbf{u}_{1}, \mathbf{u}_{2}\right) \in \mathcal{C}_{3}\right\} \\
& \mathcal{G}^{+}=\left\{\left(\mathbf{u}_{1}, \mathbf{u}_{2}\right) \mid g_{1}^{+}<0, g_{2}^{+}<0 ;\left(\mathbf{u}_{1}, \mathbf{u}_{2}\right) \in \mathcal{C}_{3}\right\},
\end{aligned}
$$

$\mathcal{G}^{-}$and $\mathcal{G}^{+}$represent all squeezing and expanding force closure grasps in $\mathcal{C}_{3}$. The 2 -fingered feasible grasping region of $\mathcal{O}_{3 D}$ is the set $\mathcal{G}=\mathcal{G}^{-} \cup \mathcal{G}^{+} . \mathcal{G}$ is also symmetric with respect to the hyperplane $\mathbf{u}_{1}=u_{2}$.

A 3-D grasping energy function, $E: \mathcal{C}_{3} \rightarrow R$, which is similar to (2.9) can be defined as: 


$$
\begin{aligned}
E_{3}\left(\mathbf{u}_{1}, \mathbf{u}_{2}\right) & =\frac{1}{2} \kappa\left\|\mathrm{p}_{1}-\mathrm{p}_{2}\right\|^{2} \\
& =\frac{1}{2} \kappa\left\|\mathcal{O}_{3 D}\left(\mathbf{u}_{1}\right)-\mathcal{O}_{3 D}\left(\mathbf{u}_{2}\right)\right\|^{2}
\end{aligned}
$$

Proposition 5.1 A pair of antipodal points, $p_{1}$ and $p_{2}$, on $\mathcal{O}_{3}$ corresponds to a critical point of $E_{3}$. Conversely, critical points of $E_{3}$ in $\mathcal{G}$, correspond to antipodal point pairs. The maximum of $E$ is a maximal grasp.

The proof of this proposition is completely analogous to that of Proposition 2.1. However, the optimization of $E_{3}$ is difficult to implement because $\mathcal{C}_{3}=S^{2} \times S^{2}$ cannot be parametrized by a single set of global parameters. The following 3-D object representation system overcomes this difficulty.

\section{Representation of 3-Dimensional Objects}

A 3-dimensional spherical product surface $\mathrm{s}: I_{u} \times I_{v} \rightarrow$ $R^{3}$ is defined as [1]:

$$
\begin{aligned}
\mathbf{s}(u, v) & =\mathbf{f}(u) \otimes \mathbf{g}(v) \\
& =\left[f_{1}(u) g_{1}(v), f_{1}(u) g_{2}(v), f_{2}(u)\right]
\end{aligned}
$$

where $f(u)$ and $g(u)$ are 2-D curves:

$$
\begin{aligned}
& f(u)=\left[f_{1}(u), f_{2}(u)\right], \\
& u \in I_{u} \equiv\left[u_{0}, u_{1}\right] \\
& \mathbf{g}(v)=\left[g_{1}(v), g_{2}(v)\right], \\
& v \in I_{v} \equiv\left[v_{0}, v_{1}\right]
\end{aligned}
$$

$f(u)$ and $g(v)$ were parametric trigonometric curves in [1]. To represent a richer set of objects, we extend this definition to use B-spline curves, as in Section 4. Let f : $I_{u} \equiv I_{m} \rightarrow R^{2}$ be an open cubic B-spline curve of $m$ segments and $\mathrm{g}: I_{v} \equiv I_{n} \rightarrow R^{2}$ be a closed cubic Bspline curve of $n$ segments. To guarantee smoothness, $f(u)$ and $g(v)$ must satisfy the following conditions:

(R-1) $f(u)$ and $g(v)$ must be regular curves.

(R-2) The curve $f(u)$ intersects the $y$-axis at $f(0)$ and $f(m)$ only. The tangents $f^{\prime}(0)$ and $f^{\prime}(m)$ must have zero slope.

(R-3) The tangent vector and the position vector of a point on $\mathbf{g}(v)$ are not parallel, i.e. $\mathbf{g}(v) \neq$ $\gamma \mathbf{g}^{\prime}(v)$ for some $\gamma \neq 0$, or $g_{1} g_{2}^{\prime}-g_{1}^{\prime} g_{2} \neq 0$.

While the spherical product maps $I_{m} \times I_{n}$ onto a surface diffeomorphic to $S^{2}$, it is not a 1-to-1 mapping because the two polar points, $p_{n}=s(0, v)$ and $p_{s}=$ $s(m, v), v \in I_{n}$, have degenerate curve forms. However, it can be shown [5] that the normal vectors at the polar points are well defined and continuous in the neighborhoods of $p_{n}$ and $\mathbf{p}_{\mathbf{s}}$.

Thus we define a 3-D object function based on spherical product surfaces:

Definition 6.1 The 3-D object function $\mathcal{O}_{3 D}^{*}: I_{m} \times$ $I_{n} \rightarrow R^{3}$ is defined to be the spherical product of an $m$-segment open cubic B-spline curve $f(u)$ and an $n$ segment closed cubic B-spline curve $g(v)$ satisfying (R$1,2,3)$.

$$
\mathcal{O}_{3 D}^{*}(u, v)=\mathbf{f}(u) \otimes \mathbf{g}(v)
$$

Let $\mathbf{p}_{1} \equiv \mathcal{O}_{3 D}^{*}\left(u_{1}, v_{1}\right)$ and $\mathbf{p}_{2} \equiv \mathcal{O}_{3 D}^{*}\left(u_{2}, v_{2}\right)$ denote the finger contact positions. The finger contact space becomes $\mathcal{C}_{3}^{*}=I_{m} \times I_{n} \times I_{m} \times I_{n}$. A grasp configuration in $\mathcal{C}_{3}^{*}$ is denoted by $u \equiv\left(u_{1}, v_{1}, u_{2}, v_{2}\right)$. Let $\mathcal{O}_{3 D u}^{*}$ and $\mathcal{O}_{3 D v}^{*}$ denote the surface tangent at point $\mathbf{p}=\mathcal{O}_{3 D}^{*}\left(u^{*}, v^{*}\right)$ along $u$ and $v$ directions respectively. The normal vector at $p$ can be defined by:

$$
\mathbf{n}= \pm \frac{\mathcal{O}_{3 D_{u}}^{*} \times \mathcal{O}_{3 D_{v}}^{*}}{\left\|\mathcal{O}_{3 D_{u}}^{*} \times \mathcal{O}_{3 D_{v}}^{*}\right\|}
$$

except at $p_{n}$ and $p_{s}$. The sign of $n$ depends on the directions of parametrization of curves $f(u)$ and $g(v)$. The unit outward normal vectors at polar points are $\mathbf{n}_{P_{n}}=[0,0,1]$ and $n_{P_{s}}=[0,0,-1]$. Since the forms of the unit normal vectors are different between polar points and other points on the object, we divide the finger contact space $\mathcal{C}_{3}^{*}=\left\{\left(u_{1}, v_{1}, u_{2}, v_{2}\right) \mid 0 \leq u_{i} \leq\right.$ $\left.m, 0 \leq v_{i} \leq n, i=1,2\right\}$ into six subsets to determine the feasible grasping region:

$$
\begin{aligned}
\mathcal{C}_{31}^{*}= & \left\{\mathbf{u} \mid 0<u_{i}<m, 0 \leq v_{i} \leq n, i=1,2\right\}, \\
\mathcal{C}_{32}^{*}= & \left\{\mathbf{u} \mid u_{1}=0,0<u_{2}<m, 0 \leq v_{i} \leq n, i=1,2\right\}, \\
\mathcal{C}_{33}^{*}= & \left\{\mathbf{u} \mid u_{1}=m, 0<u_{2}<m, 0 \leq v_{i} \leq n, i=1,2\right\}, \\
\mathcal{C}_{34}^{*}= & \left\{\mathbf{u} \mid u_{2}=0,0<u_{1}<m, 0 \leq v_{i} \leq n, i=1,2\right\}, \\
\mathcal{C}_{35}^{*}= & \left\{\mathbf{u} \mid u_{2}=m, 0<u_{1}<m, 0 \leq v_{i} \leq n, i=1,2\right\}, \\
\mathcal{C}_{36}^{*}= & \left\{\left(0, v_{1}, 0, v_{2}\right),\left(0, v_{1}, m, v_{2}\right),\left(m, v_{1}, 0, v_{2}\right),\right. \\
& \left.\left(m, v_{1}, m, v_{2}\right) \mid 0 \leq v_{i} \leq n, i=1,2\right\} .
\end{aligned}
$$

$\mathcal{C}_{31}^{*}$ represents all two-finger grasps except at the polar points. $\mathcal{C}_{32}^{*}$ and $\mathcal{C}_{33}^{*}$ represent grasp configurations where finger 1 is located at $p_{n}$ or $p_{s}$ while finger 2 is located anywhere except at the polar points. $\mathcal{C}_{34}^{*}$ and $\mathcal{C}_{35}^{*}$ are similar to $\mathcal{C}_{32}^{*}$ and $\mathcal{C}_{33}^{*}$ with finger 1 and finger 2 switching roles. $\mathcal{C}_{36}^{*}$ represents four grasps: finger 1 and 2 both at $p_{n}$ or $p_{s}$ (which we typically would ignore due to physical limitations), finger 1 at $p_{n}$ and finger 2 at $p_{s}$, finger 1 at $p_{s}$ and finger 2 at $p_{n}$.

The feasible grasping subregions, $\mathcal{G}_{i}^{*}$, in each subset $\mathcal{C}_{3 i}^{*}$ are derived by substituting (6.5), $\mathbf{n}_{P_{n}}$, and $n_{P_{\text {, }}}$ into (2.2-2.5). The feasible grasping region in $\mathcal{C}_{3}^{*}$ is the union of the feasible grasping subregions in each subset, i.e., $\mathcal{G}^{*}=\bigcup_{i=1}^{6} \mathcal{G}_{i}^{*}$ where $\mathcal{G}_{i}^{*} \subset \mathcal{C}_{3 i}^{*} i=1, \cdots, 6$.

We can formulate the modified 3-D grasping energy function, $E_{3}^{*}: C_{3}^{*} \rightarrow R$ as:

$$
\begin{aligned}
& E_{3}^{*}\left(u_{1}, v_{1}, u_{2}, v_{2}\right)=\frac{1}{2} \kappa\left\|p_{1}-p_{2}\right\|^{2} \\
& =\frac{1}{2} \kappa\left\|\mathcal{O}_{3 D}{ }^{*}\left(u_{1}, v_{1}\right)-\mathcal{O}_{3 D^{*}}\left(u_{2}, v_{2}\right)\right\|^{2} .
\end{aligned}
$$

The critical points of $E_{3}^{*}$ in $\mathcal{G}^{*}$ are antipodal points. Similarly, the maximal grasp can be defined as the global maximum of $E_{3}^{*}$. The constrained optimization or continuity methods discussed in Section 3 are applied to each subregion, $\mathcal{C}_{3 i}^{*}$ separately.

Example 2: The spherical product surface $f(u) \otimes g(v)$ is shown in fig. 6. The results of using TRUST in each of the subregions of $\mathcal{C}_{3}^{*}$ is listed in Table 2 and the corresponding grasps are shown in fig. 7. We list extremums with $u_{1}<u_{2}$ only. 


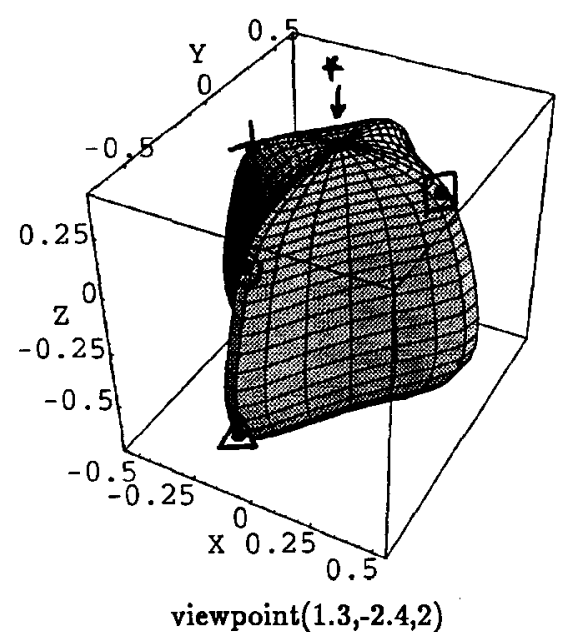

Figure 6: A 3-D object function

\begin{tabular}{|r|c|c|}
\hline \multicolumn{3}{|c|}{ Table 2: Global and Local Extrema } \\
\hline Global & $\left(u_{1}, v_{1}, u_{2}, v_{2}\right)$ & $(1.39,0.07,2.95,4.00)$ \\
Max & $P_{1}(x, y, z)$ & $(0.01,0.48,0.21)$ \\
& $P_{2}(x, y, z)$ & $(-0.07,-0.64,-0.48)$ \\
\hline Local & $\left(u_{1}, v_{1}, u_{2}, v_{2}\right)$ & $(1.44,4.00,2.98,0.07)$ \\
Max & $P_{1}(x, y, z)$ & $(-0.06,-0.55,0.20)$ \\
& $P_{2}(x, y, z)$ & $(0.01, .56,-0.49)$ \\
\hline Local & $\left(u_{1}, v_{1}, u_{2}, v_{2}\right)$ & $(1.10,2.01,3.16,6.07)$ \\
Max & $P_{1}(x, y, z)$ & $(0.37,-0.05,-0.27)$ \\
& $P_{2}(x, y, z)$ & $(-0.44,-0.01,-0.56)$ \\
\hline Local & $\left(u_{1}, v_{1}, u_{2}, v_{2}\right)$ & $(1.10,6.07,3.16,2.00)$ \\
Max & $P_{1}(x, y, z)$ & $(-0.38,0.01,0.28)$ \\
& $P_{2}(x, y, z)$ & $(0.44,0.06,-0.59)$ \\
\hline Local & $\left(u_{1}, v_{1}, u_{2}, v_{2}\right)$ & $(0.0,0.0,4.0,2.0)$ \\
Max & $P_{1}(x, y, z)$ & $(0.0,0.0,0.4)$ \\
& $P_{2}(x, y, z)$ & $(0.00 .0-0.7)$ \\
\hline
\end{tabular}

\section{Conclusion}

This paper considered antipodal point grasping of arbitrarily shaped smooth 2-D and 3-D objects. The analysis was simplified by introducing an object function which mapped a finger contact space to the object surface. The object function and the force closure grasp conditions were used to identify the feasible grasping regions of both squeezing and expanding grasps in the finger contact space. A simple grasping energy function was introduced, and it was shown that all antipodal points on the object correspond to the critical points of the energy function in the feasible grasping regions. This approach can be used with any object whose boundary can be described by continuous functions. In this paper we introduced a particular modelling method based on B-spline curves and an extension of spherical product surfaces. These modelling techniques can exactly model or closely approximate a wide variety of man-made and naturally occurring physical objects. Further, these B-spline modelling

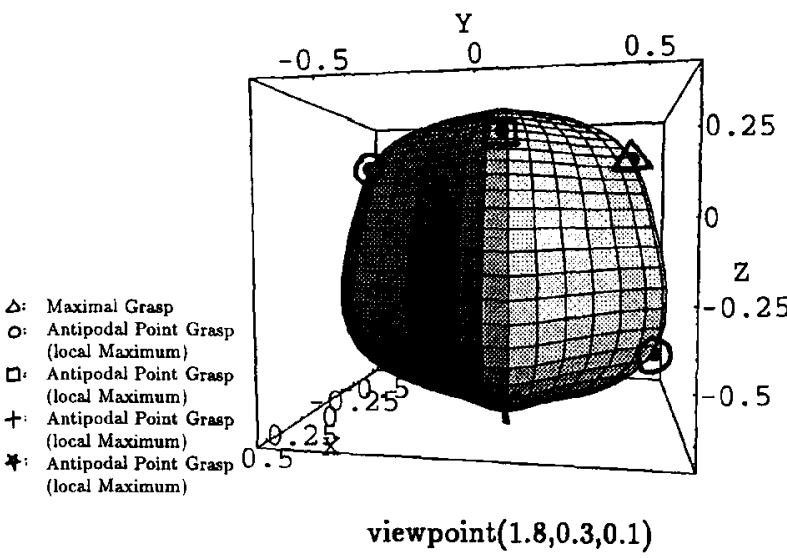

Figure 7: The max grasp and antipodal point grasps on a 3-D object

methods are well suited to the generation of object models from computer vision data. However, other modelling schemes can be similarly used.

\section{References}

[1] A. H. Barr. "Superqusdrics and Angle-Preserving Transformations," IEEE Computer Graphics and Applications. 1 p.11-23, Jan., 1981.

[2] D.P. Bertsekas, Constrained Optimization and Lagrange Multiplier Methods, NY, Academic Press, 1982.

[3] H. A. Bremermann, "A method of unconstrained global optimization," Mathematical Biosciences, 9, p. 1, 1970.

[4] B. Cetin, J. Barhen, J. Burdick, "Terminal Repeller SubEnergy Tunneling (TRUST) for Fast Global Optimization," to appear in J. Optimization Theory and Applications.

[5] I. Chen, J. Burdick. "Finding Antipodal Point Grasps on Irregularly Shaped Objects," submitted to IEEE Trans. Robotics and Automation.

[6] B. Faverjon, J. Ponce. "On Computing Two-Finger ForceClosure Grasps of Curved 2D Objects," IEEE Init. Conf. on Robotics and Automation, p424-429, Sacramento, CA, 1991.

[7] J. Hong, G. Lafferriere, B. Mishra, and X. Tan. "Fine Manipulation with Multifinger Hands," IEEE Int. Conf. on Robotics and Automation, p1568-1573, Cincinatti, OH, 1990.

[8] A. Kan , G. T. Tinmer, "Global Optimization: a Survey," in New Methods in Optimization and their Industrial Uses, ed. by J. Penot, Birkhäuser Verleg, Basel, 1989.

[9] H.B. Keller, Lectures on Numerical Methods in Bifurcation Problems, NY, Springer-Verlag, 1987.

[10] X. Markenacoff, C.H. Papadimitriou. "Optimum grip of a polygon," Int. J. of Robotic Research. vol. 8, no. 2, p17-29, April, 1989.

[11] V. Nguyen. "Constructing Force-Closure Grasps," Int. J. of Robotic Research. vol, 7, No. 3, p3-16, June, 1988.

[12] Y.C. Park, G.P. Starr. "Grasp Synthenis of Polygonal Objects," IEEE Int. Conf. on Robotics and Automation, p1574-1580, Cincinatti, OH, 1990.

[13] F. Yamaguchi, Curves and Surfaces in Computer Aided Geometry Design, NY, Springer-Verlag, 1988. 